\title{
Characterization Of Enamel Surface After Orthodontic Brackets Debonding: An In Vitro Study
}

Peixoto, A.*, Mesquita, M.F.*, Costa, H.N.*, Carvalho, P.A.**, Manso, A.*

*Centro de Investigação Interdisciplinar Egas Moniz, CiiEM, Instituto Superior de Ciências da Saúde Egas Moniz, ISCSEM, Caparica, PORTUGAL

** ICEMS, Instituto Superior Técnico, TU Lisbon, Av. Rovisco Pais, 1049-001 Lisboa, PORTUGAL

Email: andrec.peixoto@gmail.com

The use of adhesive composites for orthodontic attachments bonding to enamel has become routine clinical practice [1]. Although its use has a great number of advantages some concerns remain about the effects of bracket debonding and adhesive removal on enamel surface. Since mechanical removal of remaining adhesive can induce enamel surface damage [2], the search for an efficient and safe method has resulted in a wide array of instruments for this procedure. The aim of this study is to characterize the effects on enamel surface of three different adhesive-removal methods, after bracket debonding.

Twelve human lower molars where randomly distributed among three groups $(n=4)$ where three distinct adhesive removal methods were applied: Group A - Arkansas Stone Bur Edenta®; Group B- Tungsten Carbide Bur Dekke®; Group C -Sof-Lex ${ }^{\mathrm{TM}}$ Polishing Discs. One specimen from each group was evaluated before any procedure. Ortho Classic ${ }^{\circledR}$ metallic brackets/tubes were bonded to the three remaining specimens of each group with resinous cement (Light Bond®). Brackets/tubes were then debonded and a specific adhesive removal method was applied. The teeth were analysed by SEM. Qualitative analysis of enamel surface roughness, adhesive failure mode and adhesive remnant was carried out for each specimen at 20x, 110x, 800x and 1600x magnifications.

Before bracket bonding all three specimens showed a smooth enamel surface, with perikymata lines and residual physiologic marks. After bracket debonding an interfacial adhesive fracture, between bracket base and adhesive, was observed in Groups $\mathrm{A}$ and B, i.e. all adhesive was bonded to enamel surface, whereas in Group $\mathrm{C}$ a small amount of remnant adhesive was observed in the enamel surface, corresponding to a cohesive fracture. A heterogeneous enamel surface with a horizontal pattern of scratches over all enamel surface and small amounts of remnant unpolished adhesive was observed in Group A. In Group B an uneven surface with vertical grooves containing horizontal scratches over enamel surface and unpolished small amounts of remnant adhesive was found. In Group C a homogeneous surface, with shallow scratches and polished adhesive remnants was observed.

Although none of the three methods proved capable of removing all remnant adhesive after bracket debonding, Sof-Lex ${ }^{\mathrm{TM}}$ polishing discs had the best results in terms of homogeneity and scratch size.

The authors acknowledge financial support of the Portuguese Foundation for Science and Technology through the PEst-OE/CTM-UI0084/2011 grant.

[1] Bishara, S. E., et al.,. Angle Orthod, 78, 1078-83, 2008.

[2] Pont, H. B., et al., AM J Orthod Dentofacial Orthop. 138, 387-9, 2010. 

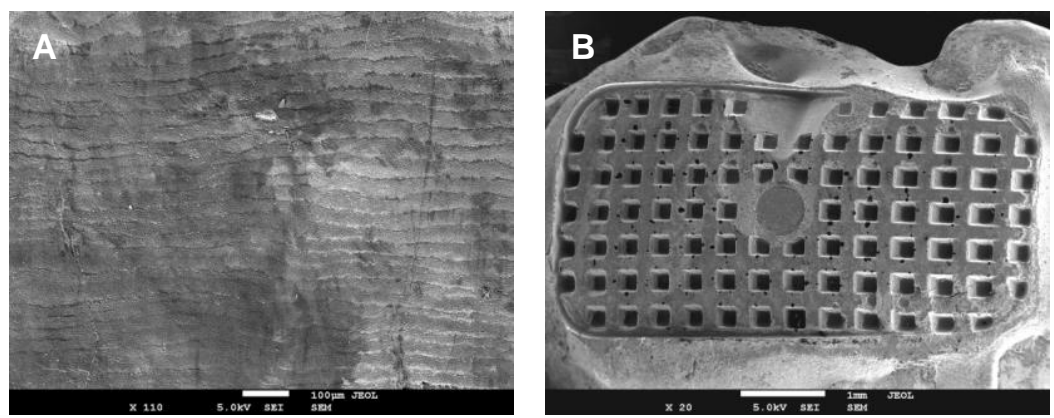

Fig. 1 - A) SEM image of Group B Enamel surface before bracket bonding: presence of perikymatas lines and physiologic scratches represents all surfaces preceding the bracket/tube. B) SEM image of Group B, of Enamel surface after bracket debonding: this image represents the most frequent adhesive failure mode where all remnants adhesive are adhered to enamel surface. Note: some air bobbles were formed during bracket/tube bonding procedure
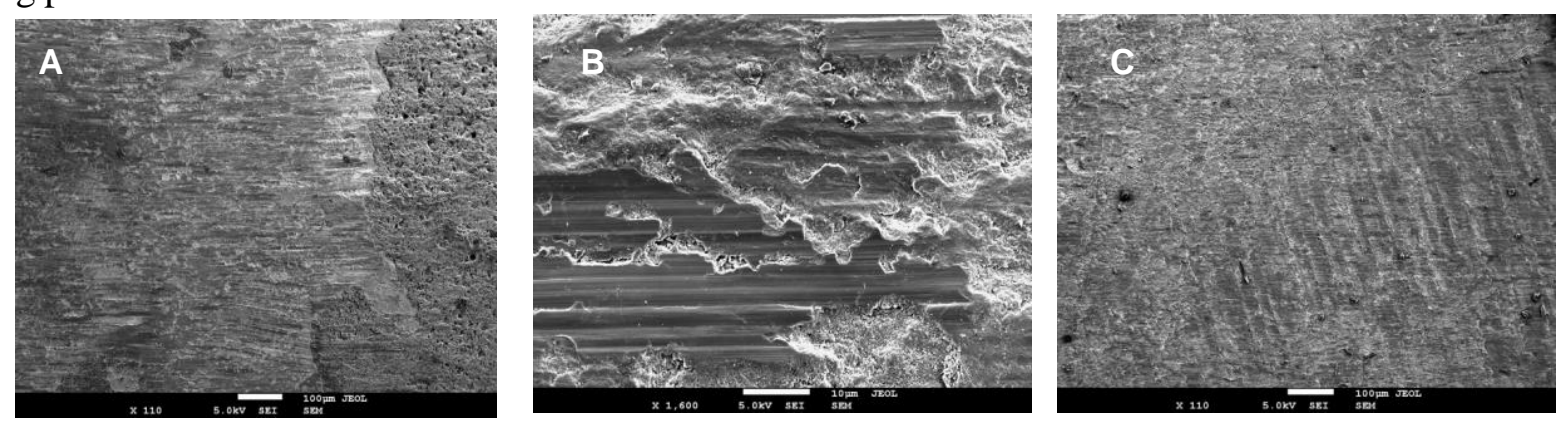

Fig. 2 - A) SEM image of Group A Enamel surface after remnant adhesive removal with Arkansas stone bur: a horizontal risked pattern is formed (left side) and some remnant adhesive is present (right side). B) SEM image of Group A Enamel surface after remnant adhesive removal with Arkansas stone bur: presence of amorphous areas due to smear layer deposition intercalated with polished remnant adhesive areas. C) SEM image of Group B Enamel surface after remnant adhesive removal with tungsten carbide bur: a rough surface with a vertical grooved pattern and is formed.
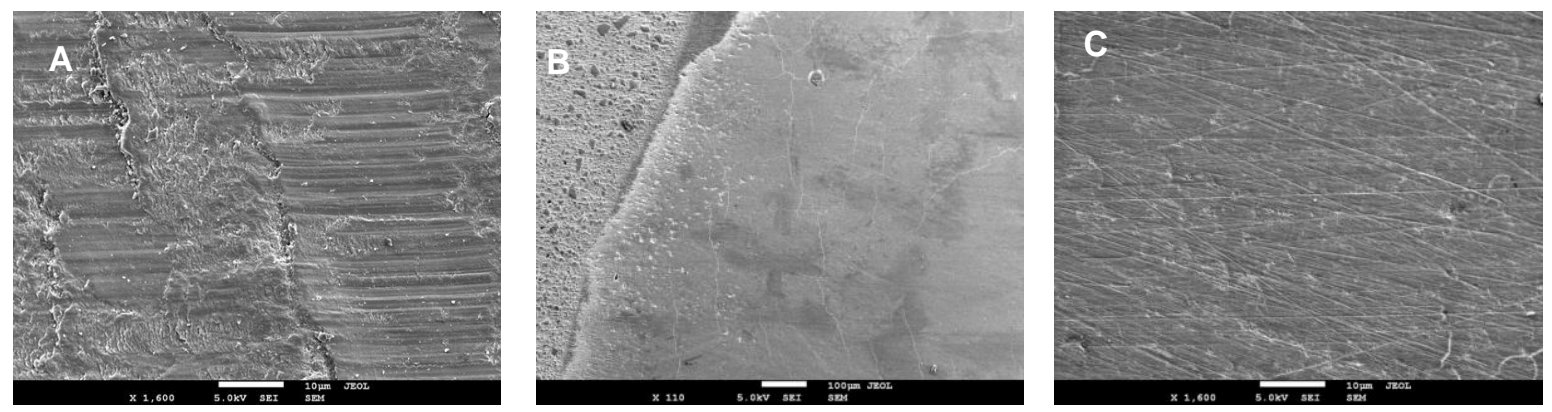

Fig.3- A) SEM image of Group B Enamel surface after remnant adhesive removal with tungsten carbide bur: horizontal risks are formed within the horizontal grooves. Some unpolished areas can be observed as a result of smear layer deposition. B) SEM image of Group C Enamel surface after remnant adhesive removal with SofLex ${ }^{\mathrm{TM}}$ polishing discs: polished remnant adhesive is present on the left side, and a thin remnant adhesive layer with an homogeneous smooth appearance is formed in areas where nearly all adhesive was removed (right side). C) SEM image of Group C Enamel surface after remnant adhesive removal with Sof-Lex ${ }^{\mathrm{TM}}$ polishing discs: polished surface is present with small risks dispersed all over the enamel. 